\title{
Epilepsy Surgery in Children versus Adults
}

\author{
Ki Hyeong Lee, M.D., ${ }^{1}$ Yun-Jin Lee, M.D., ${ }^{1,2}$ Joo Hee Seo, M.D.,' James E. Baumgartner, M.D.,' Michael Westerveld, Ph.D. \\ Comprehensive Epilepsy Center,' Advent Health for Children, Orlando, FL, USA \\ Department of Pediatrics, ${ }^{2}$ Pusan University College of Medicine, Yangsan, Korea
}

Epilepsy is one of the most common chronic neurological disorder affecting 6-7 per 1000 worldwide. Nearly one-third of patients with newly diagnosed epilepsy continue to have recurrent seizures despite adequate trial of more than two anti-seizure drugs : drug-resistant epilepsy (DRE). Children with DRE often experience cognitive and psychosocial co-morbidities requiring more urgent and aggressive treatment than adults. Epilepsy surgery can result in seizure-freedom in approximately two-third of children with improvement in cognitive development and quality of life. Understanding fundamental differences in etiology, co-morbidity, and neural plasticity between children and adults is critical for appropriate selection of surgical candidates, appropriate presurgical evaluation and surgical approach, and improved overall outcome.

Key Words : Epilepsy · Surgery · Drug resistant epilepsy · Child.

\section{INTRODUCTION}

Nearly one-third of all patients with newly diagnosed epilepsy continue to have break-through seizure despite adequate trial of more than two anti-seizure drugs : drug-resistant epilepsy $(\mathrm{DRE})^{26,33)}$. DRE is often accompanied by serious comorbidities including cognitive delay, psychosocial impairment, and drug-related side effects ${ }^{19,20}$.

Epilepsy surgery is considered to be the best treatment option if an epileptic focus is well defined and is removable without sacrificing eloquent function ${ }^{12,13,46)}$. While the basic principle remains the same regardless of age, epilepsy surgery in children requires understanding of unique neurobiological aspects of developing brain : age-dependent epilepsy syn- drome; diverse etiology; effects of chronic epilepsy and antiseizure drugs on brain development and plasticity.

\section{AGE-DEPENDENT EPILEPSY SYNDROME}

Epilepsy presents differently based on the age of patients. Focal pathology such as unilateral congenital stroke or hemimegalencephaly may present with infantile spasms in early infancy, while same etiology may present with a focal seizure in later life. This creates a challenge in terms of surgical decision as seizure semiology and electroencephalography (EEG) pattern may mimic generalized epilepsy despite apparent focal pathology. In addition, frequent epileptic seizures and epilep-

- Received : January 22, 2019 •Revised : February 22, 2019 •Accepted : February 24, 2019

- Address for reprints : Ki Hyeong Lee, M.D.

Comprehensive Epilepsy Center, Florida Hospital for Children and Florida Hospital, 615 E. Rollins Street, Orlando, FL 32803, USA

Tel : +1-513-250-5703, Fax : +1-407-303-8197, E-mail : kilee1024@gmail.com

This is an Open Access article distributed under the terms of the Creative Commons Attribution Non-Commercial License (http://creativecommons.org/licenses/by-nc/4.0) which permits unrestricted non-commercial use, distribution, and reproduction in any medium, provided the original work is properly cited. 
tic spikes in children are associated with severe cognitive delay or regression out of proportion to the severity of epilepsy, termed as epileptic encephalopathy ${ }^{20)}$.

\section{DIVERSE ETIOLOGY}

Most of the adult onset epilepsy is due to acquired pathologies such as mesial temporal sclerosis (MTS), brain tumor, stroke, central nervous system infection, and immune mediated pathologies. In children, genetic and metabolic disorders as well as developmental malformation are much more common.

Presurgical evaluation in children with DRE requires thorough a clinical exam, laboratory, and imaging tests. It is important to rule out genetic/metabolic disorders such as B6 dependent epilepsy and glucose transporter deficiency. Genetic evaluation should be considered when DRE is accompanied by cognitive and neurological deficit without clearly identified structural pathology or when there is family history of epilepsy. While ion channel mutations such as sodium voltage-gated channel alpha subunit 1 or potassium sodium-activated channel subfamily $\mathrm{T}$ member 1 mutation can often mimic focal epilepsy, surgery is not beneficial except in the form of palliative surgery such as corpus callosotomy (CC). On the other hand, not all genetic etiology should be excluded from surgery; focal cortical dysplasia (FCD) due to germ line and somatic mutation in mammalian target of rapamycin (mTOR) pathway may be amenable to surgical intervention ${ }^{4,34,36)}$.

Recent advances in immunology have allowed the diagnosis of various autoimmune encephalitides presenting with DRE. Explosive seizure onset combined with mental status change should raise suspicion for an autoimmune encephalitis such as anti-N-methyl-D-aspartate (NMDA) receptor antibody encephalitis $^{48}$. Early recognition and aggressive immune modulating therapy may be warranted.

\section{BRAIN PLASTICITY}

Epilepsy surgery is generally discouraged if epileptic focus overlaps eloquent cortices. Incomplete resection of epileptogenic zone because of the possible post-operative deficit may lead to surgical failure. In younger children (under age 6) or cognitively delayed older children, functional mapping is not feasible, making the surgical treatment of DRE more challenging. However, children tend to have better and faster recovery than adults following surgery involving eloquent cortices because of brain plasticity ${ }^{10)}$. In addition, congenital or early acquired pathology facilitate intra- or inter-hemispheric transfer of eloquent functions. These factors need to be considered in planning hemispherectomy for hemimegalencephaly or Rasmussen's encephalitis; and in determining resective margin in MRI negative DRE in children.

\section{INDICATION AND TIMING FOR PRESURGICAL EVALUATION}

In adults, epilepsy surgery is considered when patient has recurrent disabling seizures despite adequate trial of anti-seizure drugs. Patients are typically referred to tertiary epilepsy center when seizures persist after trying several anti-seizure drugs over 2-3 years or longer. In children, the presentation of DRE is often rapidly progressive. Children can have very frequent seizures from the onset and often experience status epilepticus requiring intensive care unit (ICU) admission. In addition to seizures, children with DRE sometimes present with severe developmental regression. Therefore, children with DRE can require early and urgent referral to a tertiary epilepsy center.

\section{PRESURGICAL EVALUATION}

The goal of presurgical evaluation for DRE is four-fold : 1) to confirm the diagnosis of epilepsy; 2) to find the etiology of epilepsy; 3) to localize the seizure focus; and 4) to assess the risk of neurological deficits associated with removal of a potential seizure focus.

\section{Confirmation of epilepsy and etiological work-up}

Non-epileptic psychogenic seizures are not as common as in adults. However, other non-epileptic paroxysmal disorders unique to pediatric population needs to be considered, including cardiac arrhythmia, gastroesophageal reflux, paroxysmal movement disorder, tic disorder, breath-holding spells, and sleep disorders. 


\section{Etiological work-up}

Once epilepsy is confirmed, the specific etiology of epilepsy needs to be investigated. Genetic and metabolic disorders present predominantly in early childhood. Some of these conditions can be treated with specific disease modifying treatments. For example, glucose transporter 1 deficiency or B6 dependent epilepsy can be treated with ketogenic diet or vitamin B6 respectively. In the case of an autoimmune or immune related epilepsy, immune modulating treatment should be initiated. Most importantly, only palliative surgical procedures such as corpus callosotomy should be offered to patients with genetic, metabolic, or autoimmune related epilepsies, as resective surgery is rarely effective for these types of epilepsy.

\section{Localization of seizure focus}

Presurgical evaluation in children should be customized depending on the age, developmental status, and reported seizure types, frequency, and duration. Most patients require long-term video-EEG monitoring and brain magnetic resonance imaging (MRI) with a special epilepsy protocol. When MRI and EEG do not provide adequate localizing information, fluorodeoxyglucose-positron emission tomography or single photon emission computerized tomography may be added to the evaluation. Magnetoencephalography (MEG) may reveal an irritative zone even if scalp EEG doesn't show any on account of a tangential source location.

Scalp EEG is often misleading in pediatric epilepsy. Focal or hemispheric pathology may present with generalized seizures and diffuse bilateral EEG onset ${ }^{17,511}$. Furthermore, scalp EEG can falsely lateralize seizure onset to the healthy hemisphere in cases of congenital stroke or brain tumor ${ }^{50)}$. If a hemispherectomy is considered, the decision should include MRI findings in these cases regardless of discordant EEG findings.

MRI findings of FCD can be subtle before 2 years of age as the brain is not fully myelinated. FCD may only become evident on later MRIs when myelination is more complete. FCD type I is generally less circumscribed and less impressive than type 2. Additionally, the extent of FCD is often more diffuse than is appreciated by MRI, requiring invasive EEG monitoring (IEM) and sometimes multi-lobar resection ${ }^{32)}$.

\section{Neuropscyhological evaluation}

Pediatric DRE patients have a high incidence of neurodevelopmental and mental health disorders as a co-morbidities ${ }^{49}$.
Detailed age-appropriate neuropsychological assessment should be performed in all patients if feasible before and after surgical intervention ${ }^{10}$. Comprehensive neuropsychological evaluation may be helpful to confirm localization of seizure onset in focal epilepsy, and can be used to assess the risk of cognitive morbidity associated with surgical treatment options. Advanced procedures such as functional MRI (fMRI) or intracarotid amobarbital testing may also be used to evaluate language and memory in selected older children. As such, the neuropsychology team should be actively involved in planning presurgical evaluation, determining and designing surgical procedures, and post-operative follow-up. Post-operative evaluations are important to identify the need for therapeutic interventions to address any changes that may occur after surgery. Cognitive delay or neurobehavioral disorder should not be used as justifications for deferring surgery. Successful surgery even in children with severe neurocognitive and/or behavior impairments (e.g., autism) can reduce caregiver burden and improve quality of life in the patient and caregivers. Post-operative changes in cognitive development and quality of life should be assessed as separate outcome measures in epilepsy surgery.

\section{Counseling on benefit and risk for epilepsy surgery}

The most common reason for underutilization of pediatric epilepsy surgery in developed countries is parents' fear of new neurological deficit following surgery. Unlike adults, young children or cognitively delayed young adolescents may not be eligible for non-invasive functional mapping tools such as fMRI or MEG. When a seizure focus is localized to the left hemisphere regardless of handedness, a clinician needs to discuss not only the risk of language deficits from surgery but also recovery potential due to brain plasticity. It is well accepted that language function can be transferred within or outside an epileptic hemisphere especially when the pathology is congenital or acquired shortly after birth ${ }^{6}$. This concept of brain plasticity in the developing brain applies to the epilepsy surgery itself, making a compelling argument for early surgery.

Another important aspect of preoperative counseling with the family is to set the expectation for benefits from surgery. While seizure outcome can be roughly estimated based on prognostic variables, eventual functional outcome could be quite difficult to predict even with seizure-freedom. If a primary pathology is responsible for both epilepsy and cognitive 
impairment, simply removing a seizure focus may not reverse progressive cognitive decline $e^{20)}$.

Finally, the family should be informed of the risk of uncontrolled seizures in children, including sudden unexpected death in epilepsy (SUDEP). The definition of SUDEP is "the sudden, unexpected, witnessed or unwitnessed, nontraumatic, and nondrowning death in patients with epilepsy, with or without evidence for a seizure, and excluding documented status epilepticus, in which postmortem examination does not reveal a toxicological or anatomical cause of death". The risk of SUDEP in pediatric epilepsy is estimated to be 0.90-2.22 per 1000 patient years similar to adults according to a recent study ${ }^{28)}$. Early age of onset, high seizure frequency, mental delay, multiple anti-seizure drugs, and structural abnormalities are associated with a higher risk of SUDEP').

\section{IEM}

A patient may be amenable to one step surgery without IEM when an well circumscribed lesion is corresponding to the clinical semiology and scalp EEG and its removal is not expected to create a functional deficit.

IEM is considered for the following reasons. 1) Inconclusive preoperative data (e.g., normal or nonspecific MRI scan or multiple lesions) : IEM continues to play a significant role in this patient subgroup, especially when functional imaging data are inconclusive. Removal of the entire region of seizure onset zone identified on IEM is generally required to achieve seizure freedom ${ }^{23)}$. IEM also helps clarify uncertainties of seizure origin and propagation in children with ill-defined cortical dysplasia or multiple lesions such as tuberous sclerosis ${ }^{7}$. 2) Divergent preoperative data : when divergence occurs in the context of large or deep-seated lesions, a combination of subdural and strategically placed depth electrodes is recommend$\mathrm{ed}^{24)}$. And 3) proximity of eloquent cortex : subdural EEG is preferred methods of cortical stimulation mapping over stereo-EEG (SEEG) while this preference may change with more experience in SEEG ${ }^{5)}$. Electrocorticography (ECoG)-based functional mapping has been studied to help cortical mapping in children.

Choice of IEM between subdural EEG and SEEG should be strictly based on a hypothesized epileptogenic zone and patient characteristics. Subdural EEG generally has a higher complication rate than SEEG but may be able to provide more accurate cortical mapping results especially when suspecting neocortical epilepsy ${ }^{38)}$.

SEEG is suited better for deep seated foci (insular, cingulate, orbitofrontal, inferior occipital), multiple lesions (tuberous sclerosis complex), and suspected diffuse epileptogenic network $^{37)}$. The use of SEEG has increased dramatically in the U.S. since the wide adoption of robot assisted frameless stereotaxic system ${ }^{15)}$.

Combination of subdural EEG and depth EEG may be useful in small number of patients where cortical mapping is critical but cannot ignore possible involvement of deep gray matter or poorly accessible cortical regions ${ }^{39)}$.

\section{SURGICAL PROCEDURES}

Surgical procedures for pediatric epilepsy can be broadly categorized as curative vs. palliative procedures. Curative procedures include lesionectomy, corticectomy, lobectomy, and hemispherectomy. Palliative procedures include implantation of vagus nerve stimulators, deep brain stimulators, responsive neurostimulators, and corpus callosotomy.

\section{Lesionectomy}

Removal of a pathologic lesion can be performed with or without IEM depending on the location and pathology of the lesion. For example, when scalp EEG and clinical semiology are concordant with a benign appearing tumor such as ganglioglioma or dysembryoplastic neuroepthelial tumor in nondominant hemisphere, complete resection of tumor may be recommended as the first step $\mathrm{p}^{42)}$.

\section{IEM : goal, complication and its prevention and surveillance}

Most pediatric patients require IEM to identify seizure onset zone(s), improve seizure outcome, and to limit neurological deficit following surgery. During IEM, the patient is closely monitored in an ICU setting with 24 hour a day, 7 days a week EEG technician surveillance and frequent neurological assessments. Complications of IEM such as cerebral edema, intracranial hemorrhage, and ischemic stroke may present with non-specific deterioration of mental status, which may not be easily detected without close monitoring. In addition, a pa- 
tient may develop atypical seizures different from habitual seizures during IEM because of subdural or SEEG related complications.

\section{Hemispherectomy}

Hemispherectomy is indicated if an epileptic pathology is diffusely distributed in one hemisphere and seizure-freedom is unlikely without complete disconnection of the hemisphere. Common etiologies for hemispheric epilepsy include hemimegalencephaly, diffuse hemispheric FCD, congenital large vessel stroke, Sturge-Weber syndrome, and Rasmussen's encephalitis.

Hemispherectomy in children could be challenging for a few reasons. 1) While majority of hemispheric epilepsy patients have pre-existing neurological impairment including hemiparesis and language delay, patients with preserved language and motor function may require a long period of rehabilitation following the surgery. When hemispherectomy is performed in younger patients, the rate and extent of recovery is greater than when surgery is performed in older children. 2) Despite MRI evidence of hemispheric pathology, EEG and seizure semiology can be misleading with either generalized onset or falsely lateralizing to a healthy hemisphere. For example, diffuse hemispheric FCD or hemimegalencephaly may present with infantile spasms not lateralized either by EEG or clinical semiology. While hemispherectomy of the diseased hemisphere most often leads to seizure-freedom, the possibility of bilateral seizure onset due to a somatic mutation or mosaicism needs to be clearly explained to parents before surgery. And 3) surgical failure following hemispherectomy is not uncommon and may be related to either incomplete disconnection or independent epilepsy onset from the healthy hemisphere ${ }^{9)}$. The most common areas of incomplete disconnection include basal forebrain anterior to hypothalamus, insular cortex, and corpus callosum. Intra-operative MRI with diffusion tensor imaging tractography and insular ECoG may be beneficial in ensuring that a technically adequate disconnection has been accomplished ${ }^{29,30}$. Genetic evaluation of pathologic tissue may suggest the possibility of bihemispheric pathology $y^{36)}$. Reoperation is considered only after careful reevaluation demonstrating incomplete disconnection and after seizure onset from the healthy hemisphere has been ruled out ${ }^{3}$.

\section{MRI-guided laser interstitial thermal therapy (MRgLITT)}

MRgLITT ablates an epileptogenic zone using laser generated thermal energy delivered by a stereotactically placed laser probe. The extent of thermal injury is monitored using nearreal-time MR thermography. MRgLITT can be used as a single step procedure without IEM in selected lesional cases such as MTS and cavernous hemangioma. In more complex cases, confirmation of epileptogenic zone using SEEG may improve seizure outcome. MRgLITT can be especially useful in ablating deep seated seizure focus such as MTS, hypothalamic hamartoma, insular foci, cingular foci, and periventricular heterotopic nodule ${ }^{27}$.

\section{Palliative surgery}

When an epilepsy onset zone can be identified and safely resected, an epilepsy cure can be achieved. Unfortunately, many DRE patients are not candidates for curative surgery. For those individuals, palliative surgery may be an option. To palliate is to relieve or lessen without curing. Palliative epilepsy surgical procedures are designed to reduce but not eliminate a patient's seizure burden. Any of the curative surgical procedures can be performed as a palliative procedure if patients and family agree with the idea that subsequent reduction of seizure frequency and severity may improve the quality of life. For example, lobectomy or hemispherectomy may be offered in patients with multifocal or bihemispheric epilepsy who have daily seizures despite multiple anti-seizure drugs with a clearly stated goal of improving seizure control, not seizure-freedom. The following procedures were designed to be inherently palliative regardless of epileptic syndrome : 1) corpus callosotomy (CC), 2) multiple subpial transection (MST), 3) vagus nerve stimulator (VNS), 4) deep brain stimulator (DBS), and 5) responsive neurostimulation (RNS).

CC is most effective for drop attacks - tonic/atonic/generalized tonic-clonic seizures leading to falls ${ }^{2,41,47}$. In addition, CC may lateralize seizure focus in otherwise generalized, unlocalizing epileps $y^{8,211}$. Patients with super-refractory status epilepticus have been treated with $\mathrm{CC}^{18}$. Complete $\mathrm{CC}$ is more effective than anterior 2/3 CC with no substantial differences in complication except transient disconnection syndrome ${ }^{16,22)}$. While acute disconnection syndrome was more common in complete CC group, none of the patients reported permanent disconnection syndrome. 
MST is a surgical procedure for a seizure focus overlapping eloquent cortical regions such as language and primary sensory, motor cortices. MST has been reported to have modest effect in seizure reduction ( $70 \%$ of patients with $>50 \%$ reduction and 33\% seizure-free) with few long-term deficits (6.4\%) in adults ${ }^{40)}$. In children with Landau-Kleffner syndrome, MST did not provide any additional benefit over medically treated group $^{11)}$.

VNS was originally approved as an adjunctive treatment for partial seizures in adults with DRE. However, the indication has been extended to under age 4 since 2017. VNS has modest seizure reduction : $40-60 \%$ of patient reporting $>50 \%$ seizure reduction ${ }^{44)}$. VNS is reported to reduce the incidence of status epilepticus ${ }^{14}$. VNS is less effective than CC for drop attacks, but may have synergistic effect in combination ${ }^{43}$.

DBS is an open-loop neural stimulation system targeting thalamic nuclei or hippocampus. Current approval of DBS in the U.S. is only for focal epilepsy in adults targeting anterior thalamic nucleus. However, off-label use of centromedian thalamic nucleus stimulation is reported to be effective against generalized convulsive seizures or drop attacks $\mathrm{s}^{35}$.

RNS is distinct from VNS and DBS because electrical stimulation is only delivered when electrographic seizure is detected by implanted subdural or depth electrodes (close loop stimulation). RNS is currently approved for adult DRE patients with 1-2 identified seizure foci not amenable to resective surgery due to expected functional deficit or bilateral independent seizure foci. RNS in patients with bilateral independent seizure foci provides an added benefit of chronic intracranial EEG monitoring on anti-seizure drugs which could lead to a better lateralization and subsequent resective surgery. In adults, median seizure reduction was $51-70 \%$ depending on the location of seizure focus ${ }^{25}$. There are only a few case series in pediatric DRE, but the seizure outcome seem to be equivalent to adults ${ }^{31,45)}$.

\section{CONCLUSION}

DRE in children is an enormous challenge for patients, families, and communities. Optimal treatment of pediatric epilepsy requires understanding of the unique neurobiology of pediatric epilepsy : diverse etiology, age-dependent epilepsy syndrome, and brain plasticity. Selecting a treatment option should be tailored to the patient's specific epilepsy etiology, co-morbidity, developmental status, and risk tolerance. The decision and choice of surgical intervention in pediatric DRE requires consensus of multidisciplinary epilepsy team including epileptologists, neurosurgeons, and neuropsycholgogists. The patients and their family should be fully informed of benefits and risks of surgery as well uncontrolled epilepsy. Adoption of minimally invasive surgery procedures such as robot assisted SEEG and MRgITT may increase the utilization of epilepsy surgery in children.

\section{CONFLICTS OF INTEREST}

No potential conflict of interest relevant to this article was reported.

\section{INFORMED CONSENT}

This type of study does not require informed consent.

\section{References}

1. Abdel-Mannan O, Taylor H, Donner EJ, Sutcliffe AG : A systematic review of sudden unexpected death in epilepsy (SUDEP) in childhood. Epilepsy Behav 90 : 99-106, 2019

2. Baba H, Toda K, Ono T, Honda R, Baba S : Surgical and developmental outcomes of corpus callosotomy for West syndrome in patients without MRI lesions. Epilepsia 59 : 2231-2239, 2018

3. Bartoli A, El Hassani Y, Jenny B, Momjian S, Korff CM, Seeck M, et al. : What to do in failed hemispherotomy? Our clinical series and review of the literature. Neurosurgical Rev 41 : 125-132, 2018

4. Baulac S, Ishida S, Marsan E, Miquel C, Biraben A, Nguyen DK, et al. : Familial focal epilepsy with focal cortical dysplasia due to DEPDC5 mutations. Ann Neurol 77 : 675-683, 2015

5. Britton JW : Electrical stimulation mapping with stereo-EEG electrodes. J Clin Neurophysiol 35 : 110-114, 2018

6. Brizzolara D, Pecini C, Brovedani P, Ferretti G, Cipriani P, Cioni G : Timing and type of congenital brain lesion determine different patterns of language lateralization in hemiplegic children. Neuropsychologia $40: 620$ 632, 2002

7. Brna P, Duchowny M, Resnick T, Dunoyer $C$, Bhatia S, Jayakar P : The diagnostic utility of intracranial EEG monitoring for epilepsy surgery in children. Epilepsia 56 : 1065-1070, 2015

8. Chen PC, Baumgartner J, Seo JH, Korostenskaja M, Lee KH : Bilateral 
intracranial EEG with corpus callosotomy may uncover seizure focus in nonlocalizing focal epilepsy. Seizure $24: 63-69,2015$

9. Chen S, Guan Y, Liu C, Du X, Zhang Y, Chen S, et al. : Treatment for patients with recurrent intractable epilepsy after primary hemispherectomy. Epilepsy Res 139 : 137-142, 2018

10. Cross JH, Jayakar P, Nordli D, Delalande O, Duchowny M, Wieser HG, et al. : Proposed criteria for referral and evaluation of children for epilepsy surgery: recommendations of the subcommission for pediatric epilepsy surgery. Epilepsia 47 : 952-959, 2006

11. Downes M, Greenaway R, Clark M, Helen Cross J, Jolleff N, Harkness $W$, et al. : Outcome following multiple subpial transection in LandauKleffner syndrome and related regression. Epilepsia 56 : 1760-1766, 2015

12. Dwivedi R, Ramanujam B, Chandra PS, Sapra S, Gulati S, Kalaivani M, et al. : Surgery for drug-resistant epilepsy in children. N Engl J Med 377 : 1639-1647, 2017

13. Engel J Jr, McDermott MP, Wiebe S, Langfitt JT, Stern JM, Dewar S, et al. : Early surgical therapy for drug-resistant temporal lobe epilepsy: a randomized trial. JAMA 307 : 922-930, 2012

14. Gedela S, Sitwat B, Welch WP, Krafty RT, Sogawa Y : The effect of vagus nerve stimulator in controlling status epilepticus in children. Seizure $55: 66-69,2018$

15. González-Martínez J, Bulacio J, Thompson S, Gale J, Smithason S, Najm I, et al. : Technique, results, and complications related to robot-assisted stereoelectroencephalography. Neurosurgery 78 : 169-180, 2016

16. Graham D, Tisdall MM, Gill D : Corpus callosotomy outcomes in pediatric patients: a systematic review. Epilepsia 57 : 1053-1068, 2016

17. Greiner HM, Park YD, Holland K, Horn PS, Byars AW, Mangano FT, et al. : Scalp EEG does not predict hemispherectomy outcome. Seizure 20 : 758-763, 2011

18. Greiner HM, Tillema JM, Hallinan BE, Holland K, Lee KH, Crone KR : Corpus callosotomy for treatment of pediatric refractory status epilepticus. Seizure 21 : 307-309, 2012

19. Hermann BP, Seidenberg M, Dow C, Jones J, Rutecki P, Bhattacharya A, et al. : Cognitive prognosis in chronic temporal lobe epilepsy. Ann Neurol $60: 80-87,2006$

20. Howell KB, Harvey AS, Archer JS : Epileptic encephalopathy: use and misuse of a clinically and conceptually important concept. Epilepsia 57 : 343-347, 2016

21. Hur YJ, Kang HC, Kim DS, Choi SR, Kim HD, Lee JS : Uncovered primary seizure foci in Lennox-Gastaut syndrome after corpus callosotomy. Brain Dev 33 : 672-677, 2011

22. Jalilian L, Limbrick DD, Steger-May K, Johnston J, Powers AK, Smyth MD : Complete versus anterior two-thirds corpus callosotomy in children: analysis of outcome. J Neurosurg Pediatr 6 : 257-266, 2010

23. Jayakar $P$ : Invasive EEG monitoring in children: when, where, and what? J Clin Neurophysiol 16 : 408-418, 1999

24. Jayakar P, Gotman J, Harvey AS, Palmini A, Tassi L, Schomer D, et al. : Diagnostic utility of invasive EEG for epilepsy surgery: indications, modalities, and techniques. Epilepsia 57 : 1735-1747, 2016

25. Jobst BC, Kapur R, Barkley GL, Bazil CW, Berg MJ, Bergey GK, et al. :
Brain-responsive neurostimulation in patients with medically intractable seizures arising from eloquent and other neocortical areas. Epilepsia 58 : 1005-1014, 2017

26. Kalilani L, Sun $X$, Pelgrims B, Noack-Rink M, Villanueva V : The epidemiology of drug-resistant epilepsy: a systematic review and meta-analysis. Epilepsia 59 : 2179-2193, 2018

27. Karsy M, Guan J, Ducis K, Bollo RJ : Emerging surgical therapies in the treatment of pediatric epilepsy. TransI Pediatr 5 : 67-78, 2016

28. Keller AE, Whitney R, Li SA, Pollanen MS, Donner EJ : Incidence of sudden unexpected death in epilepsy in children is similar to adults. Neurology 91 : e107-e111, 2018

29. Kim GH, Seo JH, Baumgartner JE, Ajmal F, Lee KH : Usefulness of intraoperative insular electrocorticography in modified functional hemispherectomy. BMC Neurol 17 : 162, 2017

30. Kim GH, Seo JH, Schroff S, Chen PC, Lee KH, Baumgartner J : Impact of intraoperative 3-T MRI with diffusion tensor imaging on hemispherectomy. J Neurosurg Pediatr 19 : 63-69, 2017

31. Kokoszka MA, Panov F, La Vega-Talbott M, McGoldrick PE, Wolf SM, Ghatan $S$ : Treatment of medically refractory seizures with responsive neurostimulation: 2 pediatric cases. J Neurosurg Pediatr 21 : 421427, 2018

32. Krsek P, Pieper T, Karlmeier A, Hildebrandt M, Kolodziejczyk D, Winkler $P$, et al. : Different presurgical characteristics and seizure outcomes in children with focal cortical dysplasia type I or II. Epilepsia 50 : 125137, 2009

33. Kwan P, Brodie MJ : Early identification of refractory epilepsy. N Engl J Med $342: 314-319,2000$

34. Lee JH, Huynh M, Silhavy JL, Kim S, Dixon-Salazar T, Heiberg A, et al. : De novo somatic mutations in components of the PI3K-AKT3-mTOR pathway cause hemimegalencephaly. Nat Genet 44 : 941-945, 2012

35. Li MCH, Cook MJ : Deep brain stimulation for drug-resistant epilepsy. Epilepsia 59 : 273-290, 2018

36. Lim JS, Kim WI, Kang HC, Kim SH, Park AH, Park EK, et al. : Brain somatic mutations in MTOR cause focal cortical dysplasia type II leading to intractable epilepsy. Nat Med 21 : 395-400, 2015

37. Mirandola L, Mai RF, Francione S, Pelliccia V, Gozzo F, Sartori I, et al. : Stereo-EEG: diagnostic and therapeutic tool for periventricular nodular heterotopia epilepsies. Epilepsia 58 : 1962-1971, 2017

38. Mullin JP, Shriver M, Alomar S, Najm I, Bulacio J, Chauvel P, et al. : Is SEEG safe? A systematic review and meta-analysis of stereo-electroencephalography-related complications. Epilepsia 57 : 386-401, 2016

39. Nagahama Y, Schmitt AJ, Nakagawa D, Vesole AS, Kamm J, Kovach CK, et al. : Intracranial EEG for seizure focus localization: evolving techniques, outcomes, complications, and utility of combining surface and depth electrodes. J Neurosurg 1 : 1-13, 2018

40. Ntsambi-Eba G, Vaz G, Docquier MA, van Rijckevorsel K, Raftopoulos $C$ : Patients with refractory epilepsy treated using a modified multiple subpial transection technique. Neurosurgery 72 : 890-897; discussion 897-898, 2013

41. Rahimi SY, Park YD, Witcher MR, Lee KH, Marrufo M, Lee MR : Corpus callosotomy for treatment of pediatric epilepsy in the modern era. Pedi- 
Epilepsy Surgery in Children vs. Adults | Lee KH, et al.

atr Neurosurg 43 : 202-208, 2007

42. Ramantani G, Kadish NE, Anastasopoulos C, Brandt A, Wagner K, Strobl $\mathrm{K}$, et al. : Epilepsy surgery for glioneuronal tumors in childhoodavoid loss of time. Neurosurgery 74 : 648-657; discussion 657, 2014

43. Rolston JD, Englot DJ, Wang DD, Garcia PA, Chang EF : Corpus callosotomy versus vagus nerve stimulation for atonic seizures and drop attacks: a systematic review. Epilepsy Behav 51 : 13-17, 2015

44. Serdaroglu A, Arhan E, Kurt G, Erdem A, Hirfanoglu T, Aydin K, et al. : Long term effect of vagus nerve stimulation in pediatric intractable epilepsy: an extended follow-up. Childs Nerv Syst 32 : 641-646, 2016

45. Singhal NS, Numis AL, Lee MB, Chang EF, Sullivan JE, Auguste KI, et al. : Responsive neurostimulation for treatment of pediatric drug-resistant epilepsy. Epilepsy Behav Case Rep 10 : 21-24, 2018

46. Snead OC 3rd: Surgical treatment of medically refractory epilepsy in childhood. Brain Dev 23 : 199-207, 2001
47. Stigsdotter-Broman L, Olsson I, Flink R, Rydenhag B, Malmgren K : Longterm follow-up after callosotomy--a prospective, population based, observational study. Epilepsia 55 : 316-321, 2014

48. Suleiman J, Brilot F, Lang B, Vincent A, Dale RC : Autoimmune epilepsy in children: case series and proposed guidelines for identification. Epilepsia 54 : 1036-1045, 2013

49. Vasconcellos E, Wyllie E, Sullivan S, Stanford L, Bulacio J, Kotagal P, et al. : Mental retardation in pediatric candidates for epilepsy surgery: the role of early seizure onset. Epilepsia 42 : 268-274, 2001

50. Wyllie E, Chee M, Granstrom ML, DelGiudice E, Estes M, Comair Y, et al. : Temporal lobe epilepsy in early childhood. Epilepsia 34 : 859-868, 1993

51. Wyllie E, Lachhwani DK, Gupta A, Chirla A, Cosmo G, Worley S, et al. : Successful surgery for epilepsy due to early brain lesions despite generalized EEG findings. Neurology 69 : 389-397, 2007 